\title{
Tracing the response of climate to galactic cosmic rays
}

Hiroko Miyahara ${ }^{1,2}$, Y. Yokoyama ${ }^{2,3}$, Y.T. YamaguchI ${ }^{2,3}$ AND T. NaKatsuka ${ }^{4}$

'Institute for Cosmic Ray Research, The University of Tokyo, Kashiwa, Japan; hmiya@icrr.u-tokyo.ac.jp

${ }^{2} J a p a n$ Agency for Marine-Earth Science and Technology, Yokosuka, Japan; ${ }^{3}$ Atmosphere and Ocean Research Institute, The University of Tokyo, Kashiwa, Japan; ${ }^{4}$ Graduate School of Environmental Studies, Nagoya University, Nagoya, Japan

Anomalous changes in the heliospheric environment and incident cosmic rays during the Maunder Minimum in the $17^{\text {th }}$ century may enable us to understand the impact of galactic cosmic rays on Earth's climate system.

\section{Solar activity and climate change}

The history of solar activity can be reconstructed by measuring cosmogenic nuclides such as ${ }^{14} \mathrm{C}$ in tree rings and ${ }^{10} \mathrm{Be}$ in ice cores. Comparisons between reconstructed solar activity and climate during the past 10,000 years have revealed that their variations are synchronized at a wide range of time scales, from decades to millennia. Given that the variability in solar irradiance is assumed to be small $(\sim 0.1 \%)$, it is necessary to consider other solar-related forcings to explain observed climatological phenomena such as the expansion of glaciers and changes in monsoonal activity. One possible medium of solar forcing is galactic cosmic rays modulated by the solar magnetic field in the heliosphere. The decadal and longer cycles of solar activity lead to temporal variations in the attenuation level of cosmic rays. Cosmic rays influence atmospheric ionization and the production of cloud condensation nuclei (Kirkby et al. 2011), although the detailed mechanism of their influence on the amount of clouds in the troposphere is not fully understood. Solar UV and solar wind also influence the climate by promoting chemical reactions in the stratosphere and mesosphere. The intensity of solar-related parameters follows the 11year cycle of solar activity and is more or

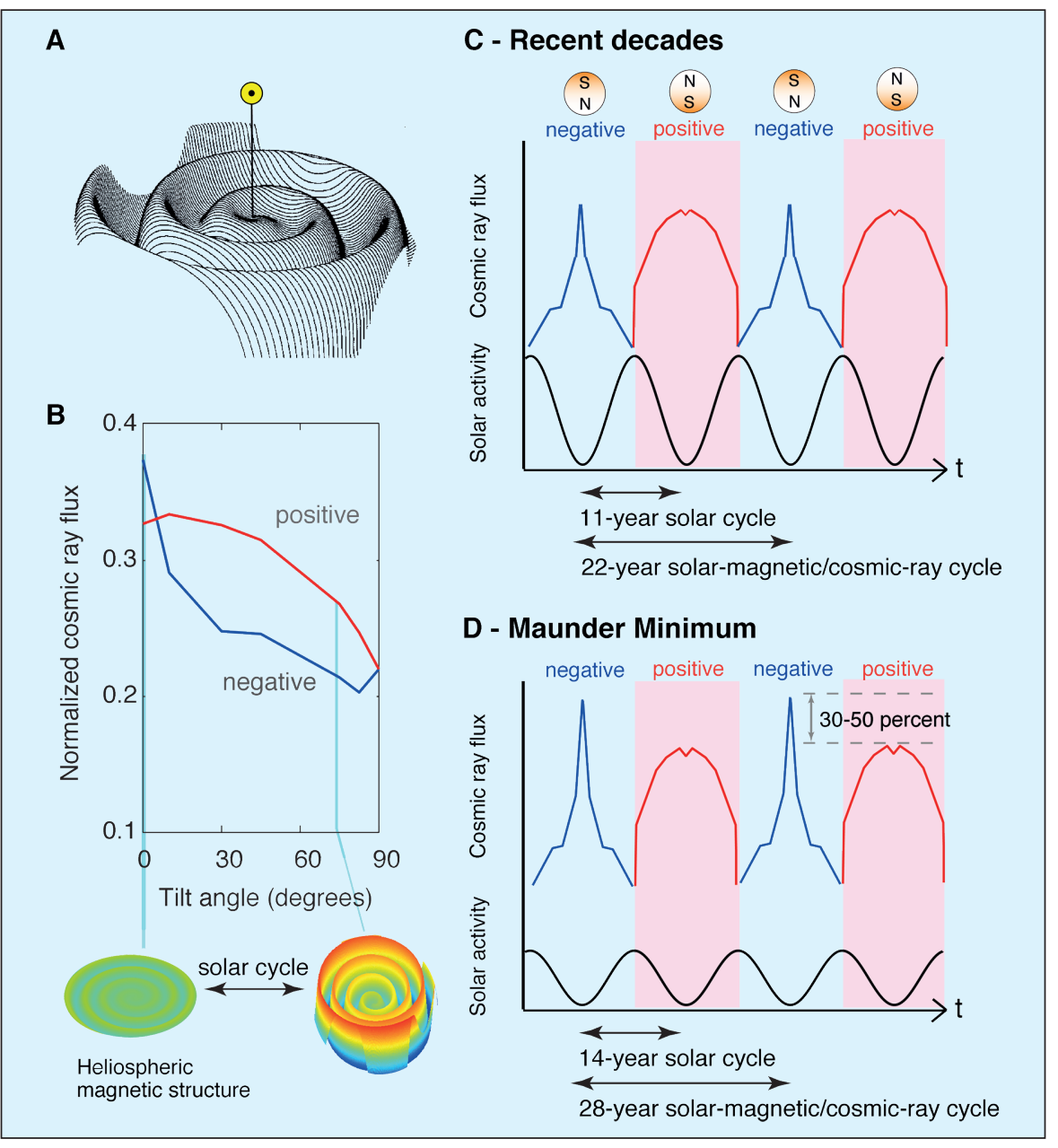

Figure 1: A) Schematic of the heliospheric magnetic field with the Sun located at the center (modified after Jokipii and Thomas 1981). B) Relation between the numerically calculated intensity of galactic cosmic rays at the Earth and the waviness of the heliospheric magnetic field (as illustrated by the schematics below the graph), for both positive and negative polarities of the solar dipole magnetic field (based on Kota and Jokipii 2001). C) Cosmic-ray time profile at the Earth in the case of a tilt angle of $5^{\circ}$ at solar cycle minima and $75^{\circ}$ at maxima, as is observed during the recent decades. $\boldsymbol{D})$ As for (C), but for tilt angles of $0^{\circ}$ at cycle minima, which reproduces the cosmic ray variation at the Maunder Minimum. Note that the polarity reverses at every maximum of the solar cycle, and that the length of the solar cycle tends to be longer (e.g. 14 years during the Maunder Minimum) when solar activity is suppressed. less synchronized among the various parameters. This makes it difficult to distinguish the climatic influence of cosmic rays versus other solar-related effects. Here we discuss the cosmic ray events around the $17^{\text {th }}$ century that might help shed some light on the influence of cosmic rays on regional and global climate.

\section{Heliospheric environment and cosmic rays}

Galactic cosmic rays are attenuated in the heliosphere, which is an expansion of the wavy solar magnetic field (Fig. 1A). The complex magnetic structure of the solar surface is swirled by the 27-day rotation of the Sun and dragged outward by the solar wind to form a spirally wound heliospheric magnetic field. The attenuation level of cosmic rays is influenced by changes in the intensity of the solar magnetic field, magnetic polarity, and the large-scale structure of the wavy heliospheric magnetic field. The waviness of the field tends to be greater when solar activity is high; consequently, it changes over the 11-year cycle of solar activity as well as at centennial to millennial time scales. The reversal in solar magnetic polarity that occurs at each decadal maximum in the solar cycle is also influencing the attenuation level of cosmic rays. The changes in the polarity of the solar magnetic dipole and in the direction of the solar magnetic field line alter the trajectory of positively charged galactic cosmic rays in the heliosphere. A numerical simulation of the intensity of cosmic rays incident to the Earth can reproduce variations in the intensity of cosmic rays due to changes in solar magnetic polarities (Fig. 1B; Kota and Jokipii 2001). In recent decades, the waviness of the heliospheric magnetic field has been suppressed to about $5^{\circ}$ at cycle minima, and has increased to about $75^{\circ}$ at cycle maxima. The observed cosmic ray variations are well reproduced by the simulated time profile in Figure 1C; however, changes in the range of tilt angles can cause anomalous variations in the time profile of cosmic rays incident to the Earth. For example, if we assume that the heliospheric magnetic field is completely flattened at 
every cycle minimum, the graph in Fig. 1B suggests that the time profile of cosmic rays would become that shown in Fig. 1D. Indeed, the record of beryllium-10 from an ice core in Greenland (Fig. 2C; Berggren et al. 2009) reveals that cosmic ray variations at the Maunder Minimum, a period of very low sunspot activity in the $17^{\text {th }}$ century, may have had the profile shown in Fig. 1D (Yamaguchi et al. 2010). The ${ }^{10} \mathrm{Be}$ record around the Maunder Minimum (AD 1645-1715) shows four anomalous spikes near minima of the solar cycle at negative polarity. The intensities of the cosmic-ray spikes are $30 \%-50 \%$ greater than those of peaks during times of positive polarity. We propose that the disappearance of sunspots at the Maunder Minimum (Fig. 2D) resulted in a completely flattened heliospheric magnetic field at solar minima, and caused anomalous enhancements in incident cosmic rays. It is also possible that maxima in long-term solar activity, such as the Medieval Solar Maximum, are characterized by anomalous temporal variations in cosmic rays.

\section{Regional and global responses to cosmic ray spikes}

The anomalous variations in cosmic rays during the Maunder Minimum enable us to trace the response of regional-global climate to galactic cosmic rays, and to understand the response of the climate system overall. It can be assumed that decadal-scale variations in solar irradiance and UV were suppressed during this 70-year period, given the absence of sunspots. In contrast, variations in cosmic rays were amplified during this period due to changes in the heliospheric environment, and four anomalous spikes occurred during this time. A comparison of the reconstructed climate and cosmic-ray-induced nuclides during the Maunder Minimum reveals that annually resolved records of climate from Greenland and Japan have synchronized 1-year-scale anomalies around the time of these four spikes (Fig. 2; Yamaguchi et al. 2010). The ${ }^{10} \mathrm{Be}$ record from the Greenland ice core has a dating error of several years, giving rise to a discrepancy in timing between the ${ }^{10} \mathrm{Be}$ record and the two climate records; however, a comparison of the superimposed spikes in ${ }^{14} \mathrm{C}$ and climate data obtained from annually-dated tree rings suggests that the climate responded to the 1-year cosmic ray spikes without a time lag. A search for these 1-year spikes in climate records (e.g. precipitation and temperature) over the globe may help to understand how cosmic rays affect the climate system.

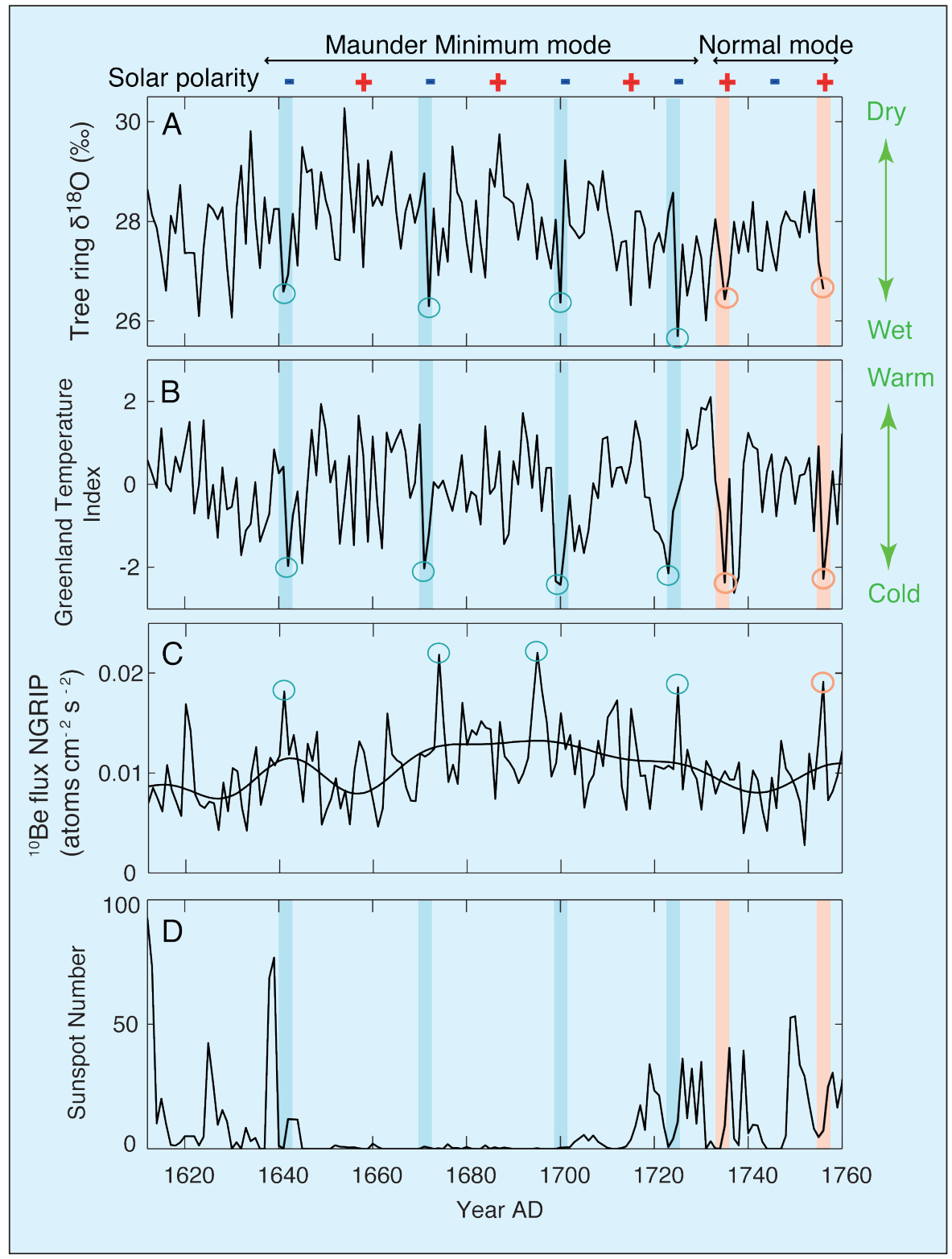

Figure 2: Temporal trends in $(\boldsymbol{A})$ relative humidity in Japan reconstructed based on ${ }^{18} \mathrm{O}$ in tree-ring cellulose (Yamaguchi et al. 2010), (B) reconstructed temperature in Greenland based on ${ }^{18} \mathrm{O}$ in ice cores (Vinther et al. 2003), (C) cosmic-ray-induced nuclides (Berggren et al. 2009), and (D) sunspot numbers (Hoyt and Schatten 1998). Open circles denote annual-scale 30\%-50\% enhancements in cosmic rays (C), and the corresponding climate response $(A, B)$. Blue and red shadings periods of negative and positive solar polarity, respectively. Figure modified from Yamaguchi et al. 2010.

\section{Changes in the 11-year solar cycle}

Frequency analyses of annually resolved ${ }^{14} \mathrm{C}$ records obtained from absolutelydated tree rings enable a reconstruction of past changes in the solar decadal cycle. The long-term record of ${ }^{14} \mathrm{C}$ reveals that solar activity has quasi-periodic variations at centennial to multi-decadal time scales, and that a long-term absence of sunspots occurs every 100-300 years. The ${ }^{14} \mathrm{C}$ records also show that the solar cycle continues uninterrupted during periods without sunspots, such as the Maunder Minimum, although the length of the cycle increases from 11 to 14 years (Fig. 1D). Inversely, the ${ }^{14} \mathrm{C}$ record indicates shorter periods in the solar cycle during maxima in long-term solar activity. During the Early Medieval Solar Maximum $\left(9^{\text {th }}-10^{\text {th }}\right.$ centuries), the length of the solar cycles decreased from 11 to $\sim 9$ years (Miyahara et al. 2008),

These results suggest that the incident cosmic rays have complex time profiles at decadal to multi-decadal scale, due to the changing solar cycle length and the heliospheric environment. This variation may help explain the complexity of multidecadal climate variations.

\section{Selected references}

Full reference list online under:

http://www.pages-igbp.org/products/newsletters/ref2012_2.pdf

Berggren AM et al. (2009) Geophysical Research Letters 36, doi: 10.1029/2009GL038004

Kota J and Jokipii JR (2001) Advances in Space Research 27: 529-534 Kirkby J et al. (2011) Nature 476(7361): 429-433

Yamaguchi YT et al. (2010) Proceedings of the National Academy of Sciences of the USA 107: 20697-20702

Miyahara H, Yokoyama Y, Masuda K (2008) Earth and Planetary Science Letters 272: 290-295 